\title{
Probability Integral Method in the Influence of Ground Settlement on a Certain Construction Land
}

\author{
Junda Lyu*, Jiacheng Gao, Chuanrong Xue \\ Key Laboratory of Disaster Prevention and Mitigation, Three Gorges University, Yichang 443002, \\ China \\ Corresponding Author.
}

\begin{abstract}
There is a potential threat of geological hazards in the construction land adjacent to a mined-out area on the west side. To accurately determine the degree of influence of the mined-out area in this area, this article has collected and sorted out five reports on a gypsum mine since 2010, combined with various parameters in the relevant reports, and used the integral probability method. Quantitatively calculate the surface displacement value, surface tilt value, absolute value of horizontal deformation and the maximum settlement value in the goaf area. The actual calculation results show that the construction land here is not affected by the goal of the gypsum mine.
\end{abstract}

Keywords: Probability integral method, land subsidence, numerical simulation, disaster prediction

\section{Introduction}

A gypsum mine is located on the west side of construction land to be assessed in Jingmen City, Hubei Province. The gypsum mine was built in the 1980s and 1990s, using the shallow hole room-pillar method, retreat mining production technology, blasting and mining, and light rail transportation. The goal of gypsum mine is unfilled [1]. There were nine ground subsidence's from 2008 to 2013. Due to historical problems, there are a large number of graves and roadways in this mining area. The shortest distance between the construction land and the goal is only $168 \mathrm{~m}$. It is necessary to determine whether the surface deformation caused by the goal of the gypsum mine will affect the construction plan of the construction land, that is, the area to be assessed.

Mine survey workers have systematically observed the process of surface movement in underground mining early on and established various forms of observation stations. The analysis and sorting of observation data have obtained the law of surface movement and given its prediction methods. The prediction methods of surface subsidence can be roughly divided into section function method, influence function method, continuum mechanics method, numerical simulation and physical model method [2]. Among these methods, the probability integration method in the influence function method is the most mature. Some scholars have used the integral probability method to quantitatively evaluate the stability of the tower foundation of the UHV transmission line in the miningaffected area of coal mines and provide the tower foundation deformation prediction value for the tower foundation structure design. Good results have been achieved in the survey and design of the UHV transmission line effect. To grasp the law of mining subsidence in the urban planning area of Jining and provide a basis for the urban planning of Jining, some scholars have collected and sorted out the observation station data of the Daizhuang Mine in the urban planning area and the data of 20 observation lines of 13 coal mines. The correlation between the parameters of the probability integration method of each mine and the mining depth, mining height, surface soil thickness and full mining degree are analysed. To solve the damage degree of surface subsidence and subsidence caused by mining and the impact on the surface land, some scholars combined the mining subsidence research theory caused by coal mining, applied the integral probability method, established the basic mathematical model, selected reasonable calculation parameters, and passed This method accurately calculates the relationship between underground mining and ground subsidence. Practice shows that this algorithm has a good use-value.

In addition to the above results, "Code for Geotechnical Investigation of Goaf Area in Coal Mines" (GB 510442014), "Technical Rules for Design and Construction of Goaf Area Highways" (JTG/TD31-03-2011) and "Buildings, Water Body, this method is recommended in the Railway and Main Roadway Coal Pillar Setting and Coal Mining Regulations, and it has successfully guided the mining of many mining areas and the treatment of surface subsidence [3]. Therefore, this paper takes the west side of construction land in Jingmen as the scope of evaluation and research, and at the same time sorts out and analyses the adequate measured data of a gypsum

ISSN: 0010-8189

(C) CONVERTER 2020

www.converter-magazine.info 
mining area over the years to obtain the settlement law of the mined-out area and whether it will affect the construction land.

\section{Regional Overview}

Figure 1 shows the 12 mined-out areas closest to the gypsum mining area and the assessment area. No. 1, 2 and 4 are ordinary mined-out areas, No. 3 are top-level mined-out areas, and 5-12 are filled mined-out areas. Among them, the shortest distance between the No. 1 goaf and the west side of Rundu construction land is about $168 \mathrm{~m}$, which is the most likely to have an adverse geological impact on the northwest side Rundu pharmaceutical construction land [4]. No. 3 mined-out area is a cap-top mined-out area, and the closest distance to the west side of the construction land is about $253 \mathrm{~m}$, which is the most likely area to have adverse geological impacts on the middle part of the west side of the construction land. The occurrence of the ore bed is gentle, with a strike of $150^{\circ}$, a tilt of $240^{\circ}$, an inclination angle of $8-10^{\circ}$, the thickness of the ore layer $X$, the gypsum layer is $10.18-10.63 \mathrm{~m}$, with an average of $10.4 \mathrm{~m}$, the buried depth is $70-150$ meters, and the average height of the goal is 8 meters. The long axis and short axis directions of the mined area of the gypsum mine have reached complete mining.

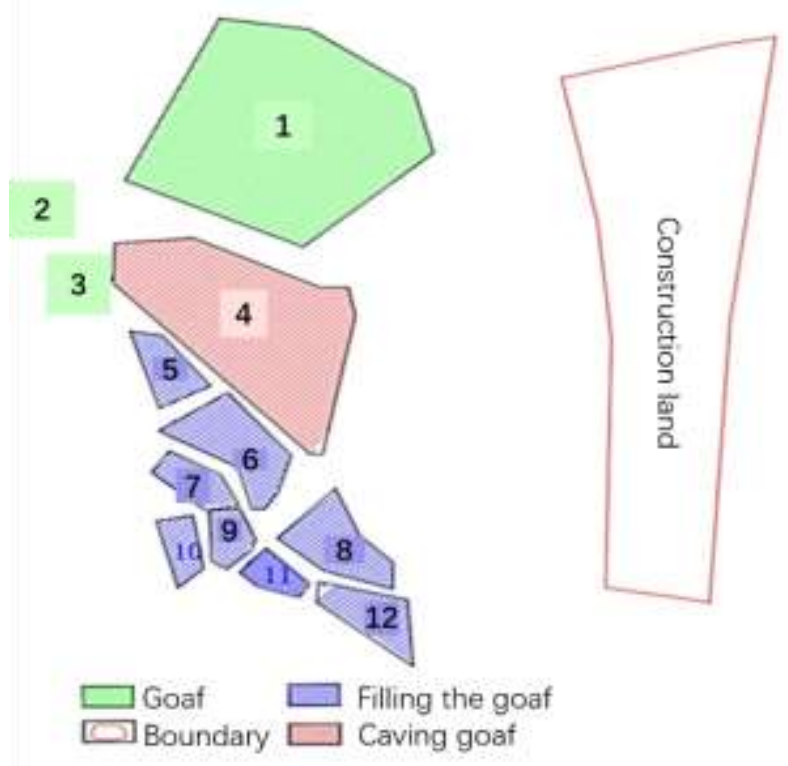

Figure 1: The distribution relationship between the assessment area and the mined-out area of a gypsum mine

\section{Research on Geotechnical Parameters of Construction Area}

Comprehensive consideration of lithology, rock layer thickness, rock quality, and its impact on the surface deformation during the gypsum mining process, the surrounding rock is classified by region. Finally, the surrounding rock and ore are mainly divided into siltstone, claystone, and gypsum rock. Three categories [5]. The physical and mechanical parameters of the rock mass in the mining area used in the paper are mainly derived from the engineering geological survey report of the mining area and referenced from similar mines, surrounding engineering geological data, and geological engineering manuals, as shown in Table 1.

Table 1 Mechanical parameters of rock mass

\begin{tabular}{|c|c|c|c|}
\hline Lithology name & Siltstone & Clay rock & Gypsum rock \\
\hline Bulk density $\rho\left(\mathrm{KN} / \mathrm{m}^{3}\right)$ & 24 & 24 & 23 \\
\hline $\mathrm{E}(\mathrm{GPa})$ & 3 & 2 & 4 \\
\hline Compressive strength $(\mathrm{MPa})$ & 22.3 & 16.6 & 27.6 \\
\hline tensile strength $(\mathrm{MPa})$ & 0.74 & 0.18 & 0.81 \\
\hline Cohesion $(\mathrm{MPa})$ & 1.86 & 0.5 & 2.8 \\
\hline$\Phi\left(^{\circ}\right)$ & 48.5 & 45 & 40 \\
\hline soften coefficient & 0.588 & 0.5 & 0.825 \\
\hline$\mu$ & 0.28 & 0.3 & 0.28 \\
\hline
\end{tabular}

ISSN: 0010-8189 
Relevant geological survey reports and research data show that the roof and floor rocks in the goaf area of the gypsum mine have good integrity. There are no joints and fissures such as faults that directly affect the calculation results. There is a lack of comprehensive surveys and structural planes for the structural planes of the study area [6]. The specific data of the parameter. Therefore, regarding the structural plane in the calculation, we only considered the strata interface. The selection of structural plane parameters is based on the parameters of geological structural planes in similar mines, as shown in Table 2.

Table 2 Stratum interface parameters

\begin{tabular}{|c|c|c|}
\hline \multicolumn{2}{|c|}{ Joint tensile strength Jtens/(N/m) } & $1.0 \times 10^{6}$ \\
\hline \multicolumn{2}{|c|}{ Joint standard stiffness JKn $/(\mathrm{N} / \mathrm{m})$} & $1.0 \times 10^{9}$ \\
\hline \multicolumn{2}{|c|}{ Joint shear stiffness JKs $/(\mathrm{N} / \mathrm{m})$} & $1.0 \times 10^{9}$ \\
\hline Joint friction angle & $\mathrm{Jf} /\left(^{\circ}\right)$ & 40 \\
\hline Joint cohesion & $\mathrm{JC} /(\mathrm{N} / \mathrm{m})$ & $1.0 \times 10^{6}$ \\
\hline
\end{tabular}

According to the relevant domestic engineering experience, the article combines the actual situation of a gypsum mine. The physical and mechanical parameters of the filling body are selected as shown in Table 3.

Table 3 Mechanical parameters of the filling body

\begin{tabular}{|c|c|}
\hline Lithology name & Filling body \\
\hline Bulk density $\rho\left(\mathrm{KN} / \mathrm{m}^{3}\right)$ & 10 \\
\hline $\mathrm{E}(\mathrm{GPa})$ & 1 \\
\hline Compressive strength $(\mathrm{MPa})$ & 22.3 \\
\hline tensile strength $(\mathrm{MPa})$ & 0 \\
\hline Cohesion $(\mathrm{MPa})$ & 0 \\
\hline$\Phi\left(^{\mathbf{}}\right)$ & 40 \\
\hline soften coefficient & 0.588 \\
\hline$\mu$ & 0.15 \\
\hline
\end{tabular}

\section{Surface Movement Prediction Method}

\subsection{Introduction to Probability Integration Method}

The theoretical basis of the influence function method is the superposition principle (Figure 2), a method between the empirical and theoretical methods.

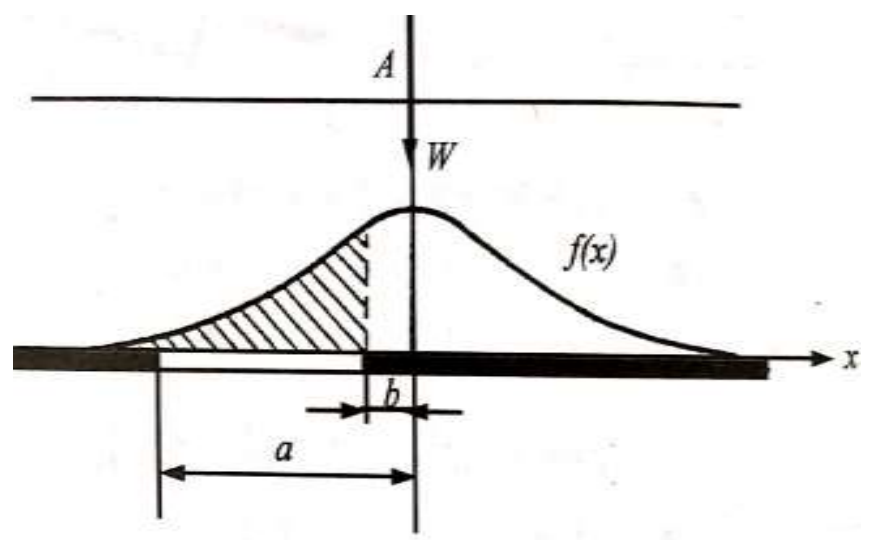

Figure 2: Superposition principle of influence function method

We assume that the mining of the ab section of the core layer produces subsidence at the surface point $\mathrm{A} W_{A}$. Divide the mining layer of section ab into $\mathrm{n}$ micro-segment mining, the mining length of each micro-segment is 
$\Delta x_{i}$, and the subsidence effect of mining on point $\mathrm{A}$ on the surface is $\Delta W_{A}^{i}$, then the total mining influence on the subsidence of point A on the surface Should be the sum of the mining impacts of each micro-segment, namely

$$
W_{A}=\sum_{1}^{n} \Delta W_{A}^{i}
$$

The impact of the mining of the small segment $\Delta x_{i} \Delta W_{A}^{i}$ is not only related to the length of the micro-segment size $\Delta x_{i}$ but also related to the position $\mathrm{x}_{\mathrm{i}}$ of the micro-segment, so there is

$$
\Delta W_{A}^{i}=f\left(x_{i}\right) \Delta x_{i}
$$

Therefore, taking $\Delta x_{i} \rightarrow 0, n \rightarrow 0$, the subsidence at point $\mathrm{A}$ of the surface of the ab section of the mining layer is

$$
W_{A}=\int_{a}^{b} f(x) d x
$$

In the formula, $\mathrm{f}(\mathrm{x})$ is the influence function. It can be seen from the nature of the integral that the sinking $W_{A}$ produced by the mining of the ab section of the ore layer at point $\mathrm{A}$ on the surface is the area enclosed by the $\mathrm{x}$ axis in the area $(a, b)$ of the influence function [7]. There are many kinds of influence functions, which can be written in the following unified form

$$
f(x)=k_{1} W_{0} g\left(r, k_{2}, x\right)
$$

In the formula, $W_{0}$ is the maximum subsidence value under full mining conditions; $\mathrm{r}$ is the primary influence radius, which is approximately the distance from the outermost point of the basin flat bottom or the maximum subsidence point to the mining boundary when thoroughly mined; $\mathrm{x}$ is the calculation point to the mining boundary the distance of the unit; $\mathrm{k}_{1}$ and $\mathrm{k}_{2}$ are the undetermined constants of the influence function [8]. The integral probability method is a method that takes the normal function as the influence function. The function is related to the mining depth, the sinking angle and the influence angle, so this method has high practical value.

As shown in Figure 3, the paper takes rectangular coordinates (x, y, z) and (s, t, m). At the mining depth $\mathrm{H}$, the ore body with a volume $2 s_{0} \times 2 t_{0} \times 2 m_{0}$ that can cause surface subsidence is mined. The sinking surface and horizontal movement surface formed on the mining surface that constitutes a unit volume of this mining are called unit sinking basin and horizontal movement basin, as shown in Figure 4. We superimpose all the unit sinking basins to form the overall sinking basin.

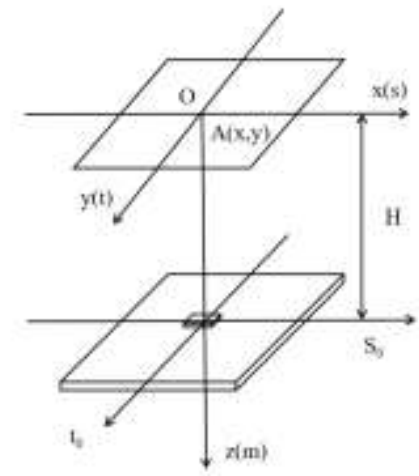

Figure. 3 Schematic diagram of mining space 


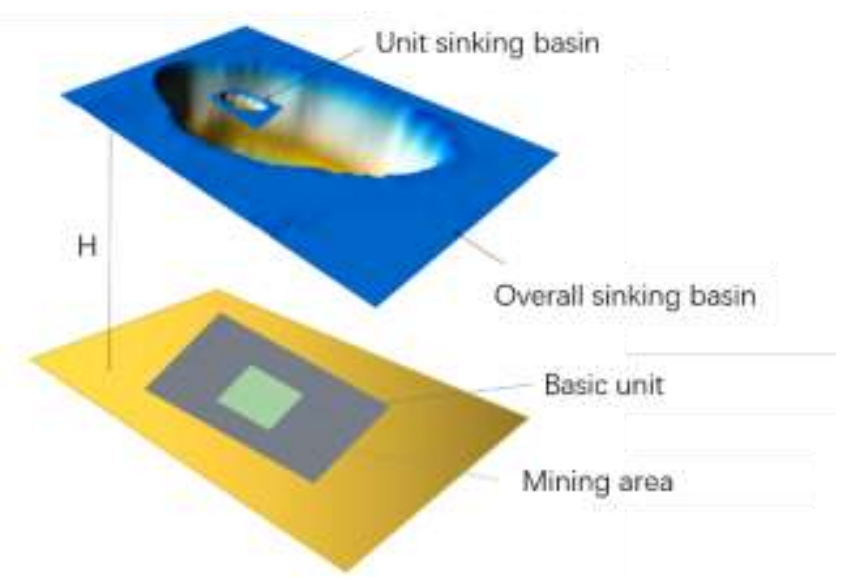

Figure 4: The relationship between the unit sinking basin and the overall sinking basin

The horizontal gypsum layer can be approximated as:

(1) The impact of micro-surface mining is isotropic. As long as the relative position of the surface and the mining microsurface remains unchanged, the mining of the microsurface with the same area has the same probability of sinking the surface point, regardless of the choice of coordinate axis [9].

(2) Attenuation of the impact of micro-surface mining. The influence of the same area on the sinking of a particular surface point decreases with the distance between the two.

(3) The volume invariance of the sinking basin in unit mining. Kochmanski believes that the final volume of the sinking basin after underground mining will be approximately equal to the volume of the production space. Therefore, the volume of the sinking basin during unit mining will eventually be equal to 1 .

(4) The increasing rate of the unit sinking basin volume is proportional to the remaining uncompacted volume. After the mining unit is mined, the roof gradually collapsed in a short time, the overlying rock mass gradually compacted and collapses. The unit sinking basin was gradually formed [10].

(5) Incompressibility of a rock mass. If the horizontal movement in the $\mathrm{x}$ and $\mathrm{y}$ directions is $\mathrm{U}, \mathrm{V}$, and the sink in the $\mathrm{z}$-direction as $\mathrm{W}$, then there is

$$
\frac{\partial U}{\partial x}+\frac{\partial V}{\partial y}+\frac{\partial W}{\partial z}=0
$$

Regardless of the time effect, the expression of the unit sinking basin in the horizontally stratified medium can be derived as

$$
\begin{gathered}
W_{e}(x, y, z)=\frac{1}{r^{2}(z)} e^{-\frac{\pi\left(x^{2}+y^{2}\right)}{r^{2}(z)}} \\
U_{e}(x, y, z)=B(z) \frac{\partial W_{e}}{\partial x}=-\frac{r(z)}{2 \pi x} \cdot \frac{d r(z)}{d z} e^{-\frac{\pi\left(x^{2}+y^{2}\right)}{r^{2}(z)}}
\end{gathered}
$$

When the ore body and the rock layer tilt, the centre of the basin moves downhill. The three-dimensional sinking unit sinking basin and the horizontally moving basin in the inclined layered medium are

$$
\begin{gathered}
U_{e}(x, y, z)=B(z) \frac{\partial W_{e}}{\partial x}+\frac{\partial \rho(z)}{\partial z} W_{e} \\
W_{e}(x, y, z)=\frac{1}{r^{2}(z)} e^{-\pi \frac{[x-\rho(z)]^{2}+y^{2}}{r^{2}(z)}}
\end{gathered}
$$

It can be seen that the horizontal movement of the unit consists of two parts: the first part is proportional to the tilt, and the second part is proportional to the sinking. $\rho(\mathrm{z})$ can be written as

$$
\rho(z)=\frac{z}{\tan \theta}
$$

Here, $\theta$ is the propagation angle of mining influence. Its equivalent effect is the angle between the middle of the basin and the middle of the mined-out area and the horizontal line on the main section of the slope, as shown in 
Figure 5. $\alpha$ is the inclination angle of the rock formation; $\mathrm{k}$ is the mining influence propagation coefficient, and the values for hard, medium-hard and weak overlying rocks are 0.7-0.8, 0.6-0.7 and 0.5-0.6, respectively [11].

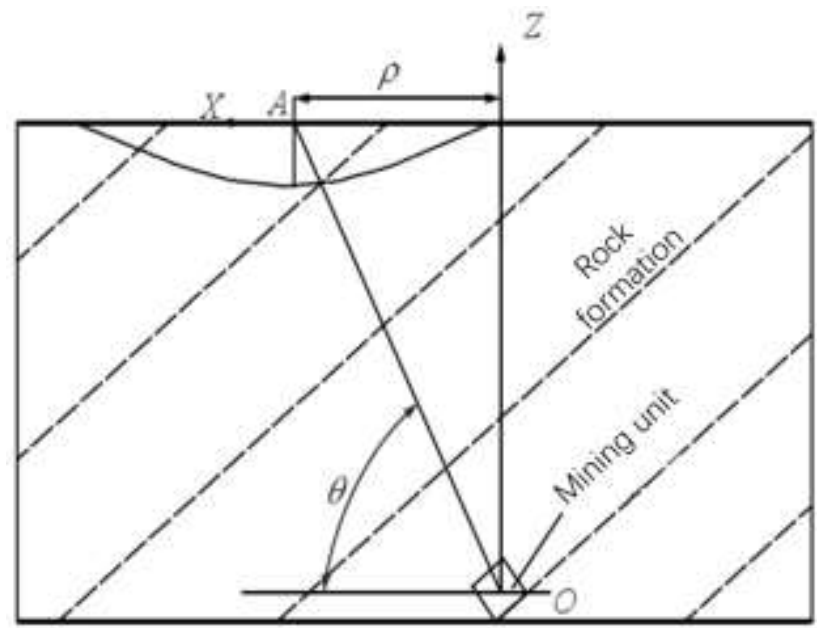

Figure 5: Schematic diagrams of the subsidence of inclined ore and rock formations

The unit surface subsidence basin calculations and horizontal displacement basin described above are all for horizontal topography. For the sloping site studied by this project, it can be considered that each point on the slope surface corresponds to a horizontal surface with the same elevation as that point, and the horizontal plane can be used to calculate the displacement corresponding to the point on the slope [12]. As shown in Figure 6, point A on the horizontal ground has the same displacement as point A on the slope. That is to say, the displacement of point A on the slope can be represented by the displacement of A when it is horizontal. In the same way, the displacement of other points on the slope can be calculated.

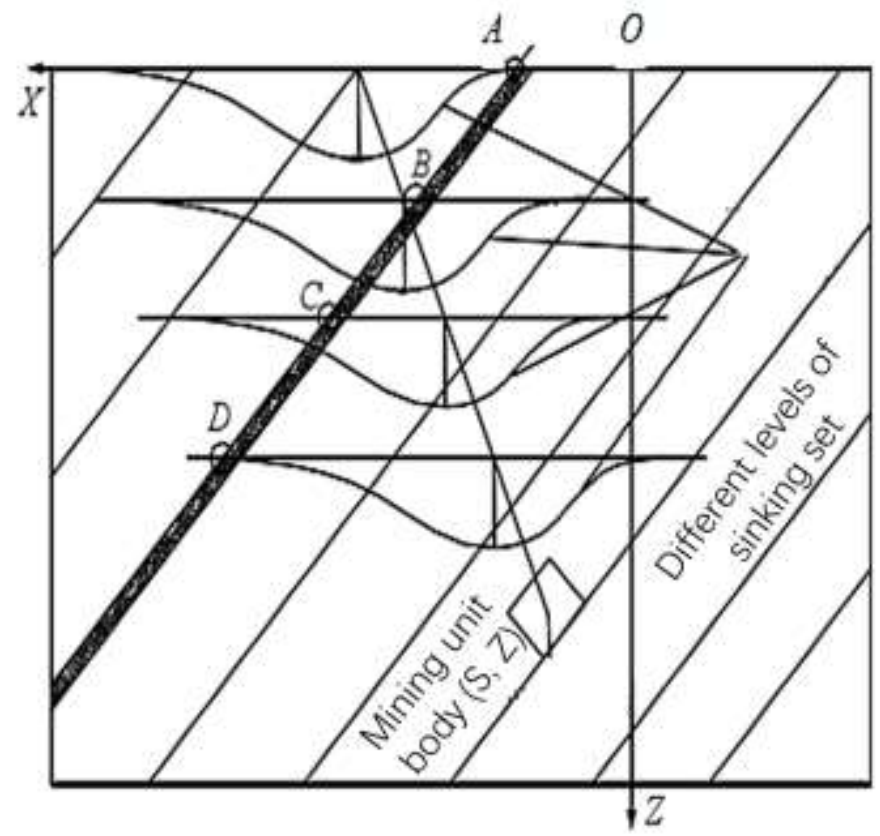

Figure 6: Solution schemes for slope terrain and surface subsidence

4.2 Determination of the relevant parameters of the probability integral method

In Section 2, the relevant rock mechanics experiments are carried out on the cores retrieved on site. The stratum characteristics and related rock mechanics parameters in the assessment area are introduced in detail. The calculation parameters of the probability integral will be introduced below:

ISSN: 0010-8189 
4.2.1 Comprehensive evaluation coefficient of overlying rock lithology

The following formula can calculate the comprehensive evaluation coefficient of the overlying rock of the ore bed

$$
P=\frac{\sum_{i=1}^{n} m_{i} Q_{i}}{\sum_{i=1}^{n} m_{i}}
$$

In the formula, mi is the standard thickness of the overlying strata.

4.2.2 Sinking coefficient

When we use the comprehensive evaluation coefficient of the overburden to determine the subsidence coefficient, we can calculate it as follows:

$$
q=0.5 \times(0.9+P)
$$

Therefore, the lithology comprehensive evaluation coefficient $\mathrm{P}$, the subsidence coefficient $\mathrm{q}$ and the lithology influence coefficient D of each ore layer corresponding to the overlying rock are calculated.

4.2.3 The main influence angle tangent

For a unit with a mining depth of $\mathrm{H}$, we can calculate the main influence angle tangent as

$$
\tan \beta=(D-0.0032 H) \cdot(-0.0038 \alpha)
$$

So, the primary influence radius can be calculated

$$
r=\frac{H}{\tan \beta}
$$

\subsubsection{Mining affects the propagation angle}

Regarding the propagation angle of mining influence $\theta_{0}$, the formulas adopted in the "Code for Geotechnical Investigation of Goaf Areas in Coal Mines" (GB 51044) and "Regulations for Coal Pillars and Coal Mining in Buildings, Water, Iron Mines and Main Roadways" are respectively:

$$
\begin{gathered}
\theta_{0}=90^{\circ}-0.68 \alpha \\
\theta_{0}=90^{\circ}-28.5^{\circ} \sin ^{2} 2 \alpha
\end{gathered}
$$

This report calculates the propagation angle of mining influence averagely based on these two formulas.

4.2.5 Calculation of horizontal movement coefficient

When mining horizontal layers, the horizontal movement coefficient $b$ does not change much, generally 0.3 . When determining the horizontal movement coefficient based on the inclination of the mining layer, the horizontal movement coefficient $b_{c}$ is calculated by the following formula.

$$
b_{c}=b \cdot(1+0.0086 \alpha)
$$

4.2.6 Knee point offset

In "Buildings, water bodies, iron mines and main shafts and coal pillars and coal mining regulations" for the soft overlying rock with uniaxial compressive strength $<30 \mathrm{MPa}$, the inflection point offset is recommended to be 0 $0.07 \mathrm{H}$, Therefore, to be conservative, the inflection point offsets of all mined-out areas in this report are taken as 0 .

4.2.7 The maximum subsidence value of the surface thoroughly mined and not fully mined is

$$
\begin{aligned}
& W_{c m}=q M \cos \alpha \\
& W_{f m}=q M n \cos \alpha \\
& n=\sqrt{n_{1} \cdot n_{2}} \\
& n_{1}=\frac{k_{1} D_{1}}{H_{0}}, n_{3}=\frac{k_{3} D_{3}}{H_{0}}
\end{aligned}
$$

In the formula, $n, n_{1}$ and $n_{3}$ are the surface mining coefficient, the trend direction mining coefficient, and the strike direction mining coefficient. When these three are more significant than 1 , all take $1 ; k_{1}$ and $k_{3}$ are the coefficients related to the overburden. The medium-soft rock takes 0.9. $D_{1}$ and $D_{3}$ are the inclination and length of the working face. 


\section{Analysis of Site Movement in the Goaf}

Since the west side of the assessment area is close to the edge of the goaf, more attention should be paid to the impact of the residual settlement of the goaf on the proposed buildings in the assessment area.

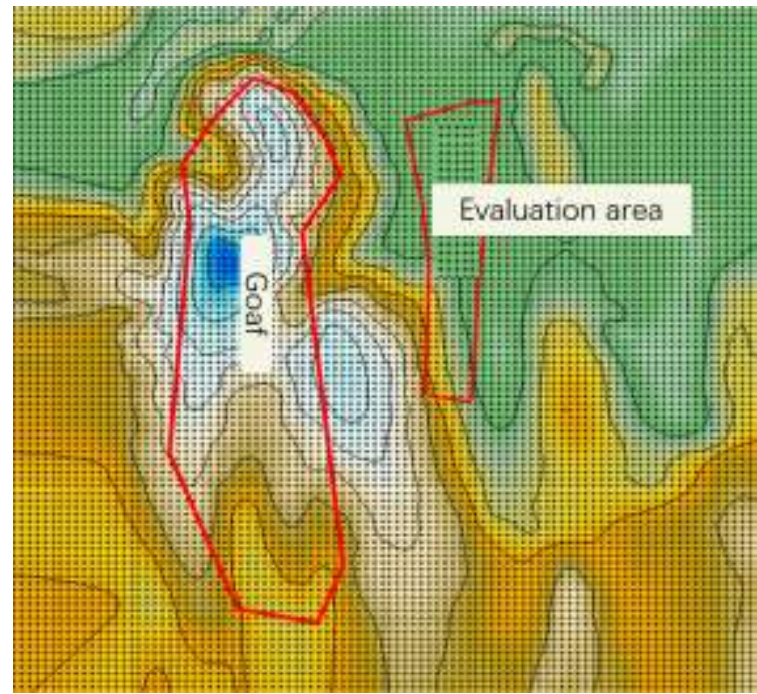

Figure 7: The model of the probability integral method to calculate the surface movement

As shown in Figure 7, the left side is the goaf area of a gypsum mine, and the right side is the evaluation area. To accurately obtain whether the subsidence of the mined-out area has an impact on the assessment area, the minedout area of $0.275 \mathrm{~km}^{2}$ on the right is triangulated. Simultaneously, to improve the calculation accuracy, the paper takes the third-order Gauss point of the triangle as the mined-out area unit. Follow the steps in section 4.1 to compile the computer program of the probability integral method to predict the surface subsidence of gypsum layer mining. The surface subsidence caused by the mined-out area will be introduced with emphasis.

Figure 8 (a) shows the surface displacement of the goaf: it is the horizontal displacement of the surface of the goaf, the maximum horizontal displacement is $1000 \mathrm{~mm}$, directly above the goaf. (b) and (c) is the absolute value of the surface inclination of the goaf and the absolute value of the horizontal deformation: the maximum value of the surface inclination in the goaf is $10 \mathrm{~mm} / \mathrm{m}$, the minimum is $1 \mathrm{~mm} / \mathrm{m}$, and the inclination value at the boundary of the goal is $3 \mathrm{~mm} / \mathrm{m}$, the maximum horizontal deformation is $6 \mathrm{~mm} / \mathrm{m}$, the minimum is $1 \mathrm{~mm} / \mathrm{m}$, and the horizontal deformation at the boundary is $2 \mathrm{~mm} / \mathrm{m}$. It can be seen that even if the residual deformation of the goaf reaches the limit state, the boundary of the goal remains low. The foundation is deformed, and the construction site is approximately $110 \mathrm{~m}$ from the boundary of the goaf. Therefore, the tilt to the horizontal deformation of the goaf does not affect the construction land. The surface settlement caused by the mining of the goal of gypsum layer: the surface settlement caused by the mining of the goal of gypsum ore is shown in 8(d), the maximum settlement in the goaf is $6000 \mathrm{~mm}$, and the settlement of the northwest and southwest corners of the construction site is $5 \mathrm{e}$ respectively $-63 \mathrm{~mm}$, and $5 \mathrm{e}-39 \mathrm{~mm}$ are numerical errors. It can be seen that the construction site will not be affected by the deformation of the goaf. 


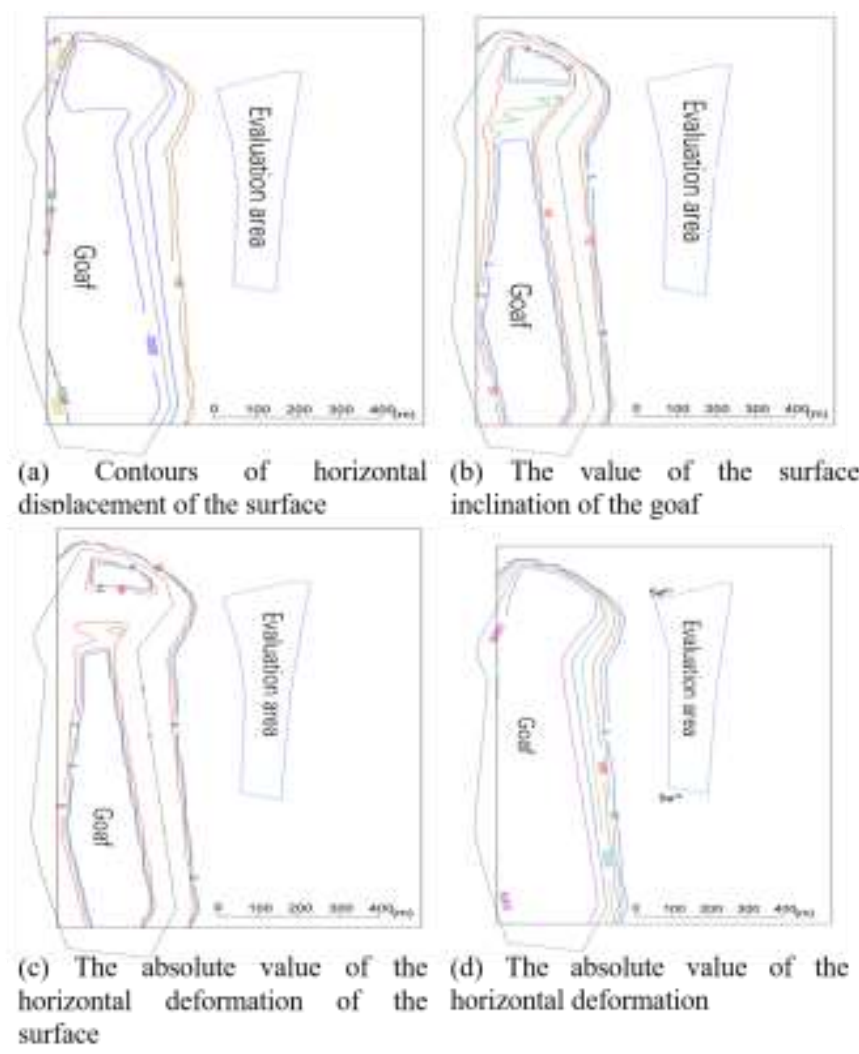

Figure 8: Schematic diagram of site movement in the goaf

\section{Conclusion}

According to the results of numerical calculation and analysis, the evaluation area close to the edge of the minedout area is not affected by the mined-out area. According to the on-site survey, no mined-out area has been found in the construction site of the assessment area. The possibility of engineering construction response or aggravation of the mined-out area collapse is slight, the degree of damage is small, and the degree of geological disasters is weak. Simultaneously, the assessment area is generally built in this area, and the risk of geological disasters that may cause or aggravate the construction after construction is assessed as small.

\section{References}

[1] M. Zheng, K. Deng, S. Du, et al. "Joint probability integral method and TCPInSAR for monitoring mining time-series deformation,” J. Indian Soc. Remote Sens, vol. 47, no. 1, pp. 63-75, 2019.

[2] Z. Liu, B.Cui, Y. Liang, et al. "Study on foundation deformation of buildings in mining subsidence area and surface subsidence prediction," Geotech. Geol. Eng. vol. 37, no. 3, pp. 1755-1764, 2019.

[3] J. Sundell, E. Haaf, J. Tornborg,et al., "Comprehensive risk assessment of groundwater drawdown induced subsidence,” Stochastic Environ. Res. Risk Assess, vol. 33, no. 2, pp. 427-449, 2019.

[4] S. Kar, E. Sen, \& S. Mukherjee, "A geospatial technique - based site suitability analysis for construction of water reservoirs in Arsha and Balarampur Blocks, Purulia,” World Water Policy, vol. 6, no. 1, pp. 52-88, 2020.

[5] J. Sundell, E. Haaf, T. Norberg, et al., "Risk mapping of groundwater - drawdown - induced land subsidence in heterogeneous soils on large areas," Risk Anal, vol. 39, no.1, pp. 105-124, 2019.

[6] C. Zhu, W. Wu, M. Motagh, et al., "Assessments of land subsidence along the Rizhao-Lankao highspeed railway at Heze, China, between 2015 and 2019 with Sentinel-1 data," Nat. Hazards Earth Syst. Sci, vol. 20, no. 12, pp. 3399-3411, 2020.

[7] P. Ding, C. Jia, S. Di, et al., "Analysis and prediction of land subsidence along significant linear engineering,” Bull. Eng. Geol. Environ, vol. 79, no. 10, pp. 5125-5139, 2020.

ISSN: 0010-8189 
[8] M. Belayneh, T. Yirgu, \& D. Tsegaye, "Effects of soil and water conservation practices on soil physicochemical properties in Gumara watershed," Upper Blue Nile Basin, Ethiopia. Ecol. Processes, vol. 8, no. 1, pp. 1-14, 2019.

[9] M. Di Ludovico, A. Chiaradonna, E. Bilotta, et al., "Empirical damage and liquefaction fragility curves from 2012 Emilia earthquake data,” Earthquake Spectra, vol. 36, no. 2, pp. 507-536, 2020.

[10] J. Englhardt, H. D. Moel, C. K. Huyck, Ruiter, et al., "Enhancement of large-scale flood risk assessments using building-material-based vulnerability curves for an object-based approach in urban and rural areas," Nat. Hazards Earth Syst. Sci, vol. 19, no. 8, pp. 1703-1722, 2019.

[11] D. K. Walkup, W. A. Ryberg, L. A. Fitzgerald, et al., "From the ground up: microhabitat use within a landscape context frames the spatiotemporal scale of settlement and vacancy dynamics in an endemic habitat specialist," Landscape Ecol, vol. 34, no. 11, pp. 2631-2647, 2019.

[12] R. Kozáková, \& A. Danielisová, "Why did they move to a barren land? Iron Age settlement and the consequences for primary woodlands in the uplands of southern Bohemia," Czech Republic. Veg. Hist. Archaeobot, vol. 29, no. 4, pp.493-507, 2020. 